rapieabbrüche sind unerwünschte Wirkungen.

Neben dem rascheren Wirkeintritt sieht Reuter den zweiten großen Vorteil der CGRP-Hemmer-Therapie dann auch in der Verträglichkeit: CGRP-Hemmer werden - zumindest auf der Grundlage der bisher verfügbaren Daten - deutlich besser toleriert als Valproat, Amitriptylin und Co. „Die Abbruchraten in den bisherigen Studien lagen in der Größenordnung von $2-4 \%$ und sind damit extrem niedrig", so Reuter. Unerwünschte Ereignisse waren in den Phase-III-Studien äußerst selten. Abgesehen von Reaktionen an der Injektionsstelle habe es in den Verumgruppen keine Ereignisse gegeben, die häufiger auftraten als in den Placebogruppen.

Die Sicherheitsdaten der Phase-IIIStudie beziehen sich bisher in der Regel auf den 3-Monats-Zeitraum. Reuter konnte aber auch eine erste Interimsanalyse einer laufenden Open-Label-
Studie zu Erenumab vorstellen, bei der 383 Patienten im Mittel für 575 Tage behandelt worden waren [Ashina $\mathrm{M}$ et al. Neurology 2017; 89(12): 1237 - 43]. Auch hier seien die Raten an Nebenwirkungen sehr niedrig: „Es gibt bisher keinen Hinweis auf einen kausalen Zusammenhang zwischen Erenumab und irgendeiner Erkrankung“, so Reuter.

\section{Exploratorische Studie zur kardiovaskulären Sicherheit}

Das trifft auch auf die kardiovaskulären Erkrankungen zu. Diesen gilt im Zusammenhang mit CGRP-Hemmern besondere Aufmerksamkeit, da CGRP einer der potentesten Vasodilatatoren ist, die dem menschlichen Organismus zur Verfügung stehen. Es gibt unter anderem die Hypothese, dass eine CGRP-Blockade mit kardioprotektiven Mechanismen interferieren könnte, die bei akuter Ischämie greifen. In diesem Kontext wurde eine explorative, randomisiert-placebo- kontrollierte Sicherheitsstudie vorgestellt, bei der 89 Patienten mit stabiler Angina pectoris einmalig $140 \mathrm{mg}$ Erenumab oder Placebo i.v. injiziert wurde. Danach erfolgte eine Belastungsuntersuchung. Die Ergebnisse waren völlig unauffällig. Weder bei der mittleren Belastungsdauer noch bei der Zeit bis zum Auftreten von ST-Senkungen $\geq 1 \mathrm{~mm}$ oder bei der Zeit bis zum Auftreten einer belastungsinduzierten Angina pectoris gab es Unterschiede zwischen Verumund Placebogruppe. Damit scheint die CGRP-Blockade in akuten Ischämiesituationen keine klinisch fassbaren negativen Effekte zu haben.

Philipp Grätzel von Grätz

U. Reuter: Anti-CGRP-treatment: small molecules and monoclonal antibodies: summary of clinical efficacy from RCTs. Plenary Session: CGRP and monoclonal antibodies, und Late-breaking-Session CGRP monoclonal antibody industry platform presentation. 18. IHC-Kongress, Vancouver/ Kanada, 8.9.2017

\title{
Hinweise auf Nutzen der nicht invasiven Hirnnervenstimulation bei Migräne
}

\author{
Es müssen nicht immer Tabletten sein: Die nicht invasive elektrische \\ Stimulation des Nervus vagus (nVNS) beziehungsweise des Nervus trigeminus \\ (eTNS) bringen sich als Alternativen zu Medikamenten bei der Akuttherapie \\ früher Migräneattacken ins Spiel.
}

$\mathrm{n}$ Vancouver wurden zu beiden Verfahren - nVNS und eTNS - randomisierte, Sham-kontrollierte Studie vorgestellt. In der von Cristina Tassorelli vom Headache Science Center der Universität Pavia koordinierten, italienischen Multicenter-Studie PRESTO kam das Gerät gammaCore (Desitin) zum Einsatz. Es wird entlang der Halsschlagadern aufgesetzt und stimuliert dort den Nervus vagus über einen Zeitraum von einigen Minuten. Das soll den nozizeptiven Reflex beeinflussen und so schmerzlindernd wirken. Patienten, bei denen die erste Behandlung nicht anschlug, wiederholten die Prozedur nach einer Viertelstunde einmalig. Primärer Endpunkt war die Schmerzfreiheit nach zwei Stunden. Dies erreichten 30,4\% der Patienten in der Stimulatorgruppe und $19,7 \%$ in der Sham-Gruppe. Die Signifikanz wurde hier knapp verfehlt.

Nach 30 Minuten und nach einer Stunde war der Unterschied allerdings signifikant, hier waren $12,7 \%$ beziehungsweise $21,0 \%$ schmerzfrei, jeweils mehr als doppelt so viel wie in der Vergleichsgruppe. Auch bei dem wichtigsten sekundären Endpunkt, dem Anteil der Patienten, die entweder schmerzfrei waren oder nur milde Beschwerden aufwiesen, wurde die statistische Signifikanz erreicht. Tassorelli sieht die PRESTO-Studie deswegen als starkes Argument, die nVNS bei akuten Migräneattacken einzusetzen.

Mit 106 Patienten etwas kleiner war eine ähnlich designte Studie zur eTNS. Dabei tragen die Patienten eine Art Bügel auf der Stirn, der von dem US-Unter- nehmen Cephaly entwickelt wurde und noch Prototypstatus hat. Der Bügel stimuliert supraorbital den Nervus trigeminus, und zwar über etwa eine Stunde, wobei der Stimulator sehr präzise gesetzt werden muss. In der Sham-Gruppe wurde ebenfalls stimuliert, aber mit niedrigerer Pulsfrequenz und Stromstärke.

Anders als die nVNS-Studie erreichte die von Dr. Denise Chou, Columbia University, koordinierte, allerdings kleinere und nur monozentrische Studie zur eTNS ihren primären Endpunkt: Der auf einer VAS von 0-10 quantifizierte Schmerz verringerte sich in der Stimulatorgruppe um 59\%, in der ShamGruppe nur um $30 \%$. Das war statistisch signifikant und der Vorteil hielt auch nach 24 Stunden noch an. Eine Verringerung der Schmerzen um mehr als die Hälfte erreichten $63 \%$ beziehungsweise $31 \%$ der Patienten.

Philipp Grätzel von Grätz

C. Tassorelli: Non-invasive vagus nerve stimulation (nVNS) for the acute treatment of migraine: a randomized controlled trial. Late breaking abstracts - Clinical and basic science. 18. IHC-Kongress, Vancouver/Kanada, 9.9.2017 